\title{
Is anticholinergic use in elderly a risk factor for frailty?
}

\author{
hasan oztin ${ }^{1}$, Yahya Ayhan Acar ${ }^{2}$, burak oymak ${ }^{3}$, and huseyin doruk ${ }^{4}$ \\ ${ }^{1}$ Izmir Ataturk Training and Research Hospital \\ ${ }^{2}$ Ankara Gulhane Egitim ve Arastirma Hastanesi \\ ${ }^{3}$ Erzurum Training and Research Hospital \\ ${ }^{4}$ Baskent University Faculty of Medicine
}

January 2, 2021

\begin{abstract}
Objective: Frailty among the elderly refers to those with limited daily activity secondary to their physical, mental, psychological and/or social limits, in addition to comorbid diseases. The use of drugs with anticholinergic burden (ACB) may lead to both unexpected adverse effects and an increased risk of frailty. In this study, we evaluated the effect of anticholinergic burden on frail patients admitted to the geriatric outpatient clinic. Materials and Methods: Included in this prospective study were 125 frail patients aged 65 and above who presented to geriatric outpatient clinics between March and November 2016. The Fried Criteria were applied for the diagnosis of frailty in patients, with five clinical properties were assessed individually in each patient. The Anticholinergic Cognitive Burden Scale was used to calculate the anticholinergic burden score for each patient. Results: A total of 125 elderly frail patients were included in the study, with a mean age of 76.74?6.67 years. The patients were composed of $77(61.6 \%)$ women and $48(38.4 \%)$ men. The effect of ACB was evaluated in the frail patients. In the frail patient group, 71 patients $(56.8 \%)$ used no drugs with ACB, while 54 patients $(43.2 \%)$ had a history of using drugs with ACB. Patients in the two frail group were evaluated based on the presence of anticholinergic burden. The mean walking speed was found to be significantly different in the two groups $(\mathrm{p}<0.001)$. The weight of the patients in ACB present and none groups were compared with a t-test, and the difference between them was found to be significant $(\mathrm{p}<0.05)$, and weight loss was found to be greater in patients with ACB present group. Conclusion: Use of drugs with an anticholinergic burden may be a risk factor for frailty by decreasing walking speed.
\end{abstract}

\section{Hosted file}

Is anticholinergic use in elderly a risk factor for frailty.pdf available at https: //authorea.com/users/386683/articles/502136-is-anticholinergic-use-in-elderly-a-riskfactor-for-frailty 